\title{
Use of computed tomography to determine the risk of patellar dislocation in 921 patients with patellar instability
}

This article was published in the following Dove Press journal:

Open Access Journal of Sports Medicine

5 March 2015

Number of times this article has been viewed

\author{
Marco Antonio Schueda' \\ Diego Costa Astur ${ }^{2}$ \\ Rodrigo Schueda Bier ${ }^{3}$ \\ Debora Schueda Bier ${ }^{4}$ \\ Nelson Astur ${ }^{5}$ \\ Moisés Cohen ${ }^{2}$ \\ 'Serviço de Pós Graduação em \\ Cirurgia do Joelho e Artroscopia \\ do IOT e Traumasports de \\ Joinville, Joinville, Santa Catarina, \\ ${ }^{2}$ Departamento de Ortopedia e \\ Traumatologia da Escola Paulista \\ de Medicina, São Paulo, ${ }^{3}$ Serviço de \\ Cirurgia do Joelho e Artroscopia \\ do IOT e Traumasports de Joinville, \\ Joinville, Santa Catarina, ${ }^{4}$ Pontifícea \\ Universidade Católica, Curitiba, \\ ${ }^{5}$ Faculdade de Ciencias Médicas da \\ Santa Casa de Misericórdia de São \\ Paulo, São Paulo, Brazil
}

\begin{abstract}
The purpose of this research was to identify reliable tomographic measurements that can detect patellofemoral abnormality and allow quantification of the risk of patellar dislocation in patients with potential patellar instability. A cross-sectional study in 921 patients with anterior pain or knee instability of at least 6 months' duration was conducted from July 2001 to December 2009. All subjects were clinically evaluated and underwent radiography and computed tomography of their knees. According to their degree of dislocating patellar dysplasia, the subjects were classified into groups for statistical comparison. There was a statistically significant difference in all measurements when the groups were compared, except for external tibial torsion angle. The most sensitive and specific measurements for determining patellar instability were the trochlear groove angle, tibial tubercle-trochlear groove distance, average patellar tilt, and average patellar height. Patients with potential patellar instability, increased tibial tubercle-trochlear groove distance, and patellar height, tilt, and deviation measurements had a greater risk for patellar dislocation. The clinical relevance of this study is to determine measurements that are able to tell us about patellar dislocation risk.
\end{abstract}

Keywords: patellofemoral instability, knee, patellofemoral syndrome

\section{Introduction}

Patellofemoral instability refers to any functional and/or anatomical abnormality within the structures surrounding the patella and the femoral trochlea leading to knee pain, patellar dislocation, subluxation, and symptoms of patellar hypermobility. ${ }^{1-18}$ It is a disabling condition that is most prevalent in younger people and seen more frequently in females than in males. ${ }^{14}$ The etiology is multifactorial, and hip rotation, tibial tuberosity positioning, femoral groove and patellar articular facet anatomy, gait biomechanics, and imbalance of the knee extensor apparatus can all lead to poor alignment of the patella. ${ }^{3}$

Radiographic studies are used to evaluate the anatomy of the patellofemoral joint, but cannot accurately assess the initial degree of knee flexion. The advent of computed tomography (CT) enables more accurate analysis of the patellofemoral joint than is possible using plain radiographs. ${ }^{4,5,7,8}$ Further, advances in image acquisition and technology such as image overlapping and dynamic imaging studies (muscle contraction/relaxation, knee extension/flexion) have led to new parameters, such as trochlear dysplasia and tibial tuberosity-trochlear groove distance, along with patellar height, tilt, and deviation. These criteria are utilized to analyze patellofemoral joint morphology and help define abnormal anatomy. ${ }^{2,9,36}$
Correspondence: Diego Costa Astur Department of Orthopedics and Traumatology, Universidade, Federal de São Paul, Rua Borges Lagoa, 783, 58 andar, Vila Clementino, São Paulo CEP 04038-032, Brazil Email mcastur@yahoo.com 
Even with multiple parameters available to analyze patellofemoral instability, it is still difficult to determine how this syndrome manifests. The Lyon orthopedic group has described four distinct groups of patients with dislocating patellofemoral dysplasia: those with painful patellar syndrome (anterior knee pain without radiographic abnormalities and previous dislocation); those with potential patellar instability (anterior knee pain with abnormal tomography but no previous dislocation), those with objective patellar instability (at least one previous confirmed patellar dislocation, reported by the patient, attending physician, or radiographic documentation, such as medial patellar facet avulsion or lateral condyle fracture); and those with major patellar instability (frequent or permanent dislocation of the patella). ${ }^{8}, 11$

The purpose of this study was to identify CT measurements that can reliably detect patellofemoral abnormality according to the Lyon definition of dislocating patellar dysplasia and would allow quantification of the risk of patellar dislocation in patients with potential patellar instability. We believe that there are some $\mathrm{CT}$ measurements that can reliably estimate the risk of dislocation.

\section{Patients and methods}

After obtaining institutional review board approval, a crosssectional study was conducted from July 2001 to December 2009. Of 2,816 knees screened, 1,792 from 921 patients were enrolled in the study. Inclusion criteria were age $18-50$ years and anterior knee pain or instability of at least 6 months' duration. Exclusion criteria included skeletal immaturity, previous ligamentous knee surgery, concurrent surgery on the contralateral limb, and major patellar instability. Forty-five asymptomatic patients were recruited as a control group and underwent the same evaluation protocol.

After clinical, radiographic, and CT evaluation, the subjects were divided in four groups according to the Lyon classification of dislocating patellar dysplasia: control subjects (group 1); those with painful patellar syndrome (group 2); those with potential patellar instability (group 3); and those with objective patellar instability (group 4). Patients with major patellar instability were excluded because of associated conditions leading to permanent or frequent dislocation, so were not within the scope of the present study.

All patients included in the study underwent $\mathrm{CT}$ of the knee. The CT studies were reviewed by the same radiologist, who measured the following parameters: femoral neck anteversion angle; knee rotation angle; malleolar condylar angle; external tibial torsion angle; tibial tubercle-trochlear groove distance (TTTG); trochlear groove angle; patellar tilt with relaxed quadriceps, patellar tilt with contracted quadriceps, and with a flexed knee; average patellar tilt (APT); patellar deviation with relaxed quadriceps (PDRQ), patellar deviation with contracted quadriceps, and with a flexed knee; average patellar deviation (APD); and average patellar height (APH) with relaxed quadriceps, patellar height with contracted quadriceps, and with a flexed knee (Figure 1). Measurement of patellar deviation, height, and tilt with a flexed knee was performed with 20 degrees of knee flexion. Patellar height was measured using the Insall-Salvati

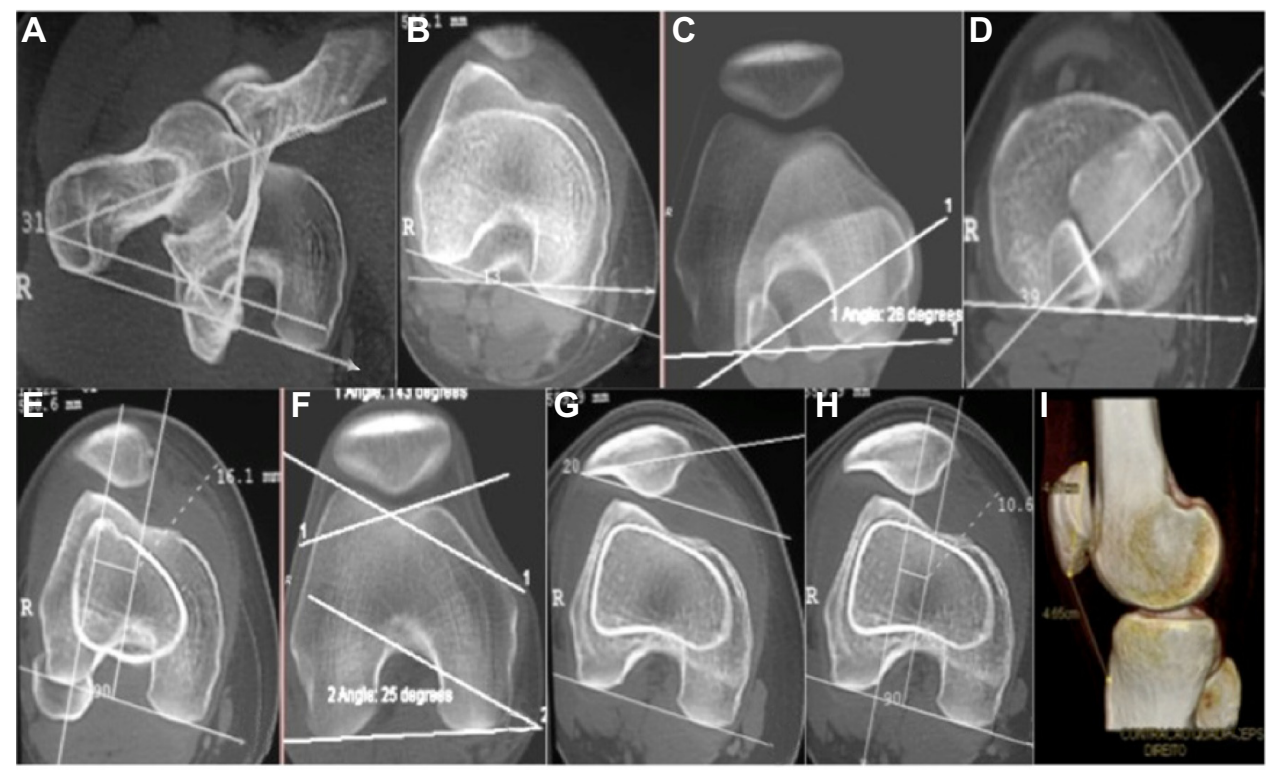

Figure I (A) Femoral neck anteversion angle, (B) knee rotation angle, (C) malleolar condylar angle, (D) external tibial torsion angle, (E) tibial tubercle-trochlear groove distance, $(\mathbf{F})$ trochlear groove angle, $(\mathbf{G})$ patellar tilt, $(\mathbf{H})$ patellar deviation, and $(\mathbf{I})$ patellar height. 
method, where patellar tendon length to the length of the patella were measured. ${ }^{26,39}$

Statistical analysis of the CT measurements was performed in order to compare group 1 with groups 2, 3, and 4 and to compare group 3 with group 4 . In addition, analyze risk of patellar dislocation in patients with potential patellar instability (group 3).

\section{Statistical analysis}

The Student's $t$-test was used to test for differences between mean values. The Mann-Whitney test was applied to variables with an asymmetric distribution. Measurements in the four predefined groups were compared using analysis of variance and the Kruskal-Wallis test. Receiver operating characteristic curves were used to estimate cutoff points and the sensitivity and specificity for each group. A multiple logistic regression model was used to identify the most significant variables for occurrence of patellar dislocation. All analyses were twotailed test. The sample size was estimated based on a type I error of $5 \%$, a type II error of $10 \%$, a minimum difference between proportions of $30 \%$, a semi-amplitude of 0.04 , and a minimum significance level of 0.05 , giving a minimum power of $90 \%$ test.

\section{Results}

The predetermined parameters were investigated by CT scan in 1,792 of 2,816 knees screened. The population comprised 921 subjects, including 363 (39.4\%) males and 558 (60.6\%) females of mean age $33.7 \pm 11.2$ years (Table 1). Patients were categorized into groups according to their knee CT measurements (see Table 2).

There was no statistically significant difference in CT measurements between controls (group 1) and subjects with painful patellar syndrome (group 2). Comparing controls (group 1) and subjects with potential patellar instability (group 3), there was a statistically significant $(P<0.05)$ difference in all measurements, except for external tibial torsion angle $(P=0.61)$. Comparing controls (group 1) and those with objective patellar instability (group 4), there was a statistically significant $(P<0.05)$ difference in all measurements, again

Table I Study demographics and clinical characteristics

\begin{tabular}{lllll}
\hline & Group I & Group 2 & Group 3 & Group 4 \\
\hline Knees (n) & 87 & 596 & 1,070 & 39 \\
Male (\%) & 31.1 & 38.7 & 41.3 & 35.3 \\
Female (\%) & 68.9 & 61.3 & 58.7 & 64.7 \\
Pain (\%) & 0 & 100 & 86.48 & 100 \\
Laxity (\%) & 0 & 7.6 & 7.6 & 61.3 \\
\hline
\end{tabular}

except for external tibial torsion angle $(P=0.61)$. Further, comparing the receiver operating characteristic curves for the controls (group 1) and those with potential patellar instability (group 3), the most sensitive and specific measurements for diagnosing potential instability were the trochlear groove angle (sensibility [S] 52\%, specificity [E] 90.8\%), TTTG (S 63\%, E 92\%), APT (S 58\%, E 79.3\%), and APH (S 63.3\%, E 59.8\%).

Measurements were compared for subjects with potential patellar instability (group 3) and those with objective patellar instability (group 4), and their risk of patellar dislocation was estimated using a multiple logistic regression model, presenting the probability of $0 \%$ to $100 \%$ occurrence of dislocation in accordance with the $\mathrm{CT}$ measurement values, presenting the probability of $0 \%$ to $100 \%$ occurrence of dislocation in accordance with the CT measurement. Knees with a markedly increased TTTG and patellar height, tilt, and deviation were noted to have a higher risk of patellar dislocation. The probability of dislocation increased with TTTG values $>15 \mathrm{~mm}$, reaching $20 \%$ ifTTTG was $>35 \mathrm{~mm}$ (Figure 2). Moreover, the risk of dislocation increased significantly with APH measurements $>1$. The risk of dislocation was about $6 \%$ when APH was 1.2 , and increased by $30 \%$ when APH was 1 (Figure 3). The risk of dislocation also increased when APT was over 30 degrees. When APT was 68.5 degrees, the risk of dislocation increased by $80 \%$ (Figure 4). When we considered the APD values, the risk of dislocation increased significantly with measurements over $10 \mathrm{~mm}$. When the APD value was $35 \mathrm{~mm}$, the risk increased by $85 \%$ (Figure 5 ).

Patellar tilt and deviation were the most significant measurements to estimate risk of potential patellar dislocation turning into an objective patellar dislocation (APT, odds ratio $1.07,95 \%$ confidence interval 1.03-1.11; APD, odds ratio $1.12,95 \%$ confidence interval 1.05-1.18; $P<0.001$ ).

\section{Discussion}

In this cross-sectional study, we noted that some patellofemoral CT measurement methods were accurate for detecting patellofemoral instability, and were different from those used in patients with anterior knee pain but without anatomical abnormalities in their extensor apparatus. A global analysis of our objective results concludes that TTTG, APT, and trochlear groove angle are the most reliable measurements for diagnosing patellar instability $(P<0.001)$.

After clinical evaluation of symptomatic knees in the present study, all patients in groups 2, 3, and 4 had pain, instability of the patellar was mostly observed in group 4 
Table 2 Mean and standard deviation computed tomography measurements for I,792 knees according to the four groups analyzed

\begin{tabular}{|c|c|c|c|c|c|c|c|c|}
\hline \multirow[t]{2}{*}{ Measurements } & \multicolumn{2}{|l|}{ Group I } & \multicolumn{2}{|l|}{ Group 2} & \multicolumn{2}{|l|}{ Group 3} & \multicolumn{2}{|l|}{ Group 4} \\
\hline & Average & SD & Average & SD & Average & SD & Average & SD \\
\hline FNAA $\left(^{\circ}\right)$ & 18.9 & 8.7 & 17.7 & 7.7 & 20.6 & 8.4 & 23.2 & 8.5 \\
\hline TGA $\left({ }^{\circ}\right)$ & 135.2 & 6.5 & 135.3 & 6.4 & 142.6 & 9.0 & 145.3 & 9.4 \\
\hline $\operatorname{KRA}\left({ }^{\circ}\right)$ & 4.7 & 3.2 & 4.1 & 3.0 & 5.4 & 3.7 & 6.8 & 4.5 \\
\hline $\operatorname{ETTA}\left({ }^{\circ}\right)$ & 32.5 & 5.8 & 31.6 & 7.2 & 31.7 & 7.7 & 32.7 & 7.7 \\
\hline $\operatorname{MCA}\left({ }^{\circ}\right)$ & 34.8 & 6.7 & 33.6 & 8.2 & 36.4 & 8.3 & 39.6 & 9.2 \\
\hline TTTG (mm) & 12.4 & 2.4 & 12.2 & 2.7 & 16.2 & 3.9 & 17.8 & 4.5 \\
\hline PTRQ & 9.6 & 3.5 & 8.7 & 3.5 & 14.1 & 5.9 & 22.2 & 9.1 \\
\hline PTCQ & 10.2 & 3.8 & 9.6 & 4.1 & 16.5 & 7.4 & 27.5 & 11.0 \\
\hline PTFK & 6.5 & 3.1 & 6.3 & 2.9 & 8.7 & 4.5 & 11.8 & 6.9 \\
\hline APT & 14.7 & 5.0 & 13.4 & 5.2 & 22.4 & 9.2 & 35.9 & 14.3 \\
\hline PDRQ & 2.0 & 2.3 & 1.8 & 2.5 & 4.1 & 3.4 & 9.7 & 6.1 \\
\hline PDCQ & 4.7 & 2.7 & 4.2 & 2.7 & 7.1 & 3.6 & 12.3 & 5.4 \\
\hline APD & 4.4 & 3.1 & 3.9 & 3.5 & 7.6 & 5.0 & 15.9 & 8.5 \\
\hline PDFK & -1.1 & 1.8 & -1.3 & 1.7 & -0.3 & 2.5 & 2.0 & 4.9 \\
\hline PHRQ & 0.88 & 0.12 & 0.89 & 0.12 & 0.95 & 0.14 & 1.02 & 0.16 \\
\hline PHCQ & 0.98 & 0.14 & 0.98 & 0.13 & 1.06 & 0.17 & 1.16 & 0.20 \\
\hline PHFK & 0.96 & 0.14 & 0.97 & 0.12 & 1.04 & 0.15 & 1.14 & 0.18 \\
\hline
\end{tabular}

Abbreviations: FNAA, femoral neck anteversion angle; KRA, knee rotation angle; MCA, malleolar condylar angle; ETTA, external tibial torsion angle; TTTG, tibial tubercletrochlear groove distance; TGA, trochlear groove angle; PTRQ, patellar tilt with relaxed quadriceps; PTCQ, patellar tilt with contracted quadriceps; PTFK, patellar tilt with a flexed knee; APT, average patellar tilt; PDRQ, patellar deviation with relaxed quadriceps; PDCQ, patellar deviation with contracted quadriceps; PDFK, patellar deviation with a flexed knee; APD, average patellar deviation; PHRQ, patellar height with relaxed quadriceps; PHCQ, patellar height with contracted quadriceps; PHFK, patellar height with a flexed knee; SD, standard deviation.

(those with objective patellar instability). The need to understand the etiology of symptoms in this group of patients and to define the most appropriate way of treating them has led a large number of investigators to look for radiographic abnormalities that could suggest a pathologic origin for this syndrome..$^{4,7,12,23,29,32,41}$ Other studies have analyzed the relationship between patellofemoral abnormalities and abnormalities of the hip, thigh, and leg, also defining measurements such as the radiographic distance between the $\mathrm{TTTG}^{5}$ and the Q-angle in axial images of the patella. ${ }^{24}$

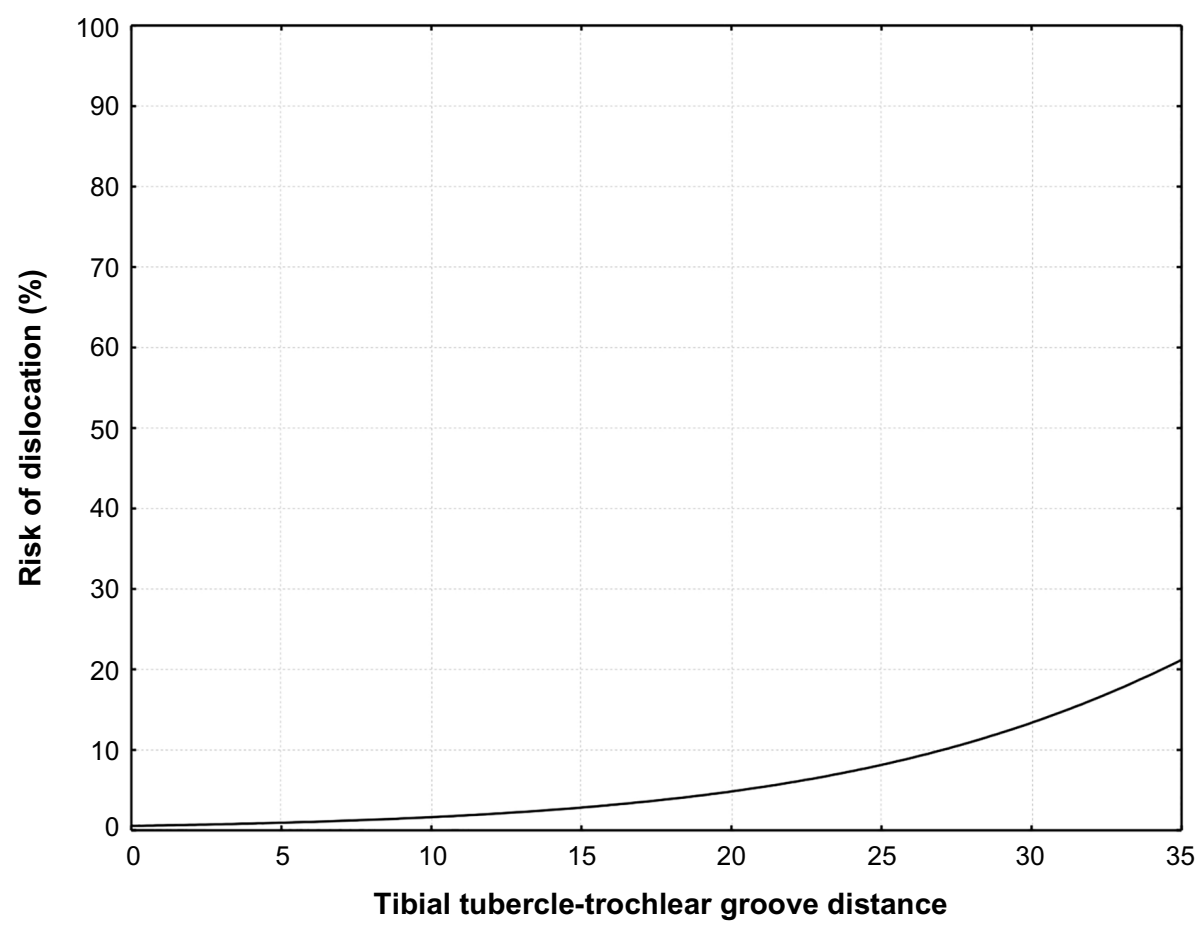

Figure 2 Risk of group 3 dislocations according to TTTG measured. The risk of dislocation increases significantly with measurements over I5 mm. When the TTTG value is $35 \mathrm{~mm}$, the risk increases by $20 \%$.

Abbreviation: TTTG, tibial tubercle-trochlear groove. 


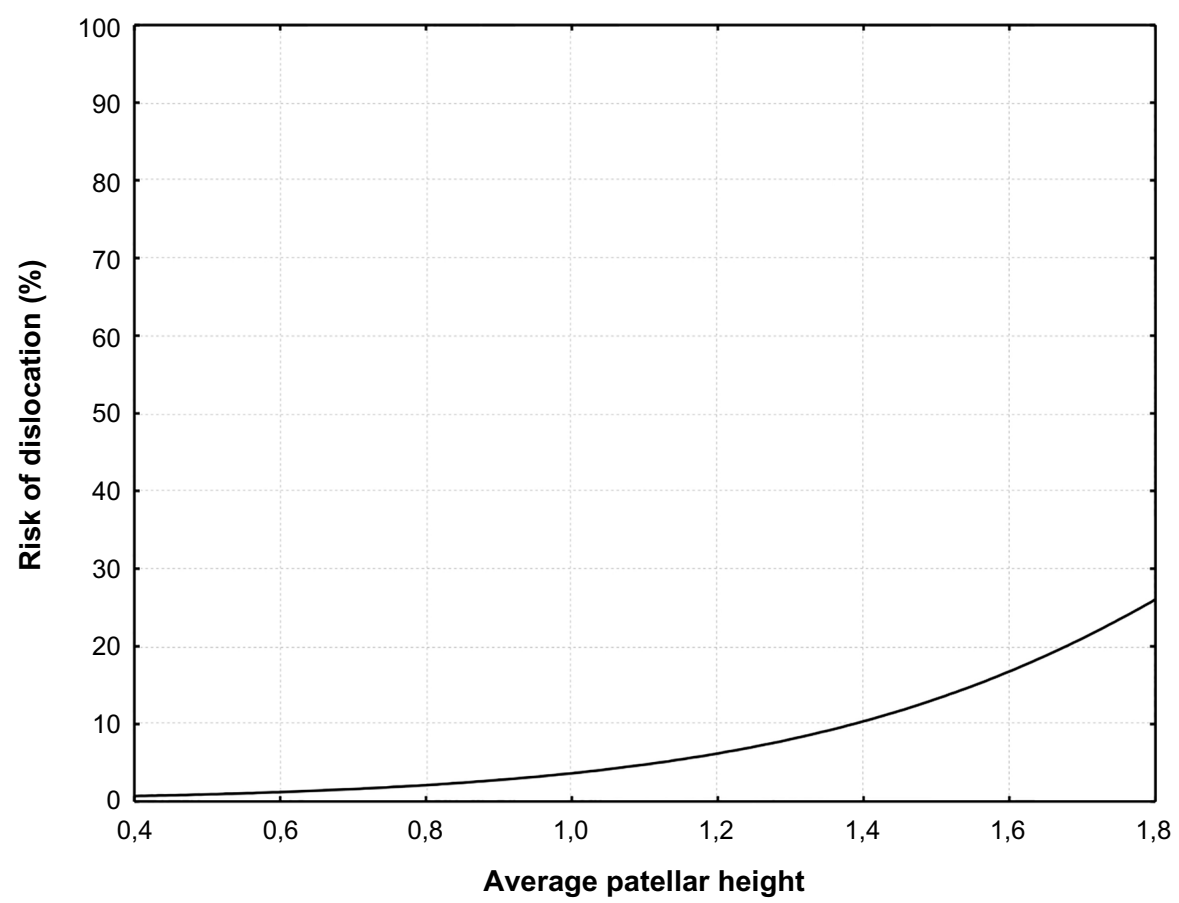

Figure 3 Risk of group 3 dislocations according to APH measured. The risk of dislocation increases significantly with measurements over I. When the APH value is I.8, the risk increases by $30 \%$.

Abbreviation: APH, average patellar height.

When CT scans were first used to study the patellofemoral joint, a better understanding of this syndrome became possible because of the creation of highly sensitive and specific images with no distortion, as well as the ability to overlap images and measure rotational parameters in the lower limbs. A large number of authors have reported the superiority of this imaging modality when analyzing the patellofemoral joint in knee extension, which is impossible with X-rays..$^{26,25,31}$ Galland et al ${ }^{16}$ established normal and abnormal values for knee measurements in radiographs

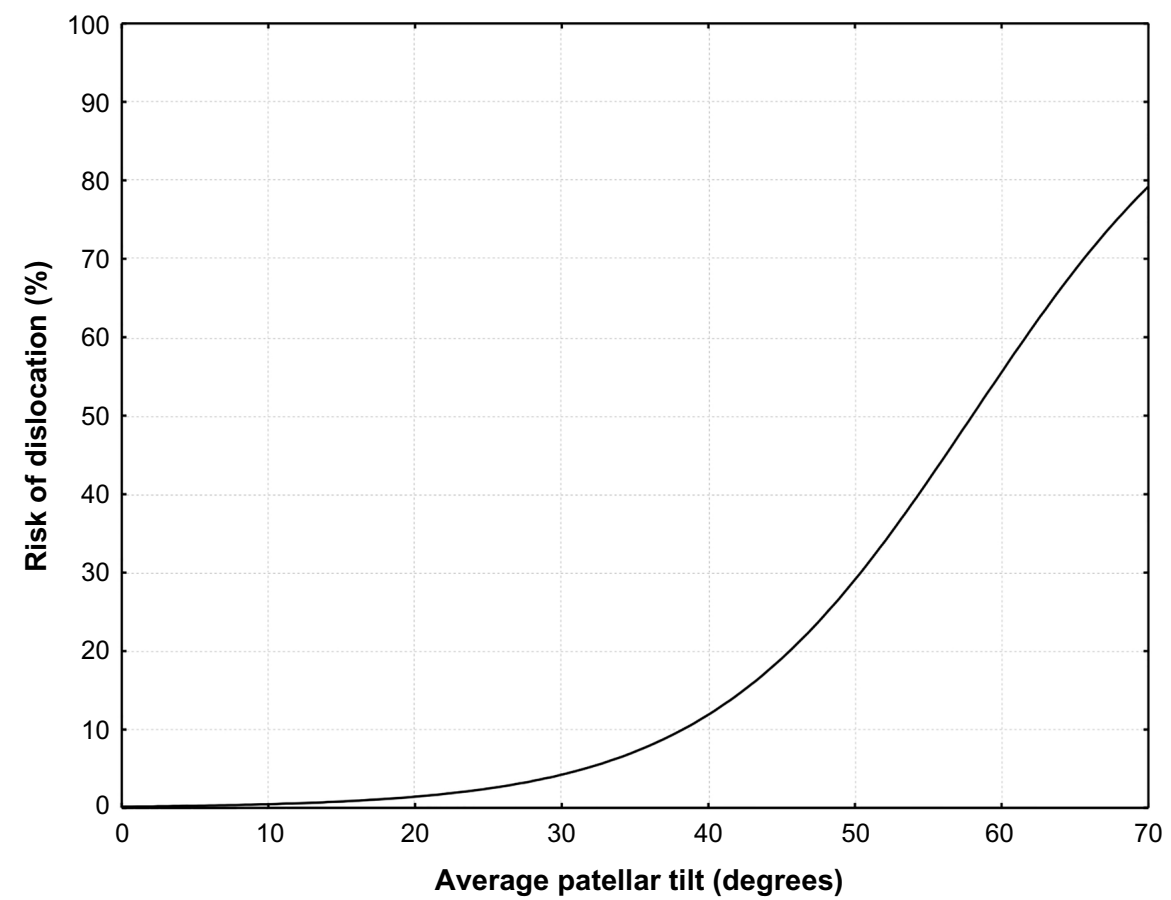

Figure 4 Risk of group 3 dislocations according to APT measured. The risk of dislocation increases significantly with measurements over $30^{\circ}$. When the APT value is $68.5^{\circ}$, the risk increases by $80 \%$.

Abbreviation: APT, average patellar tilt. 


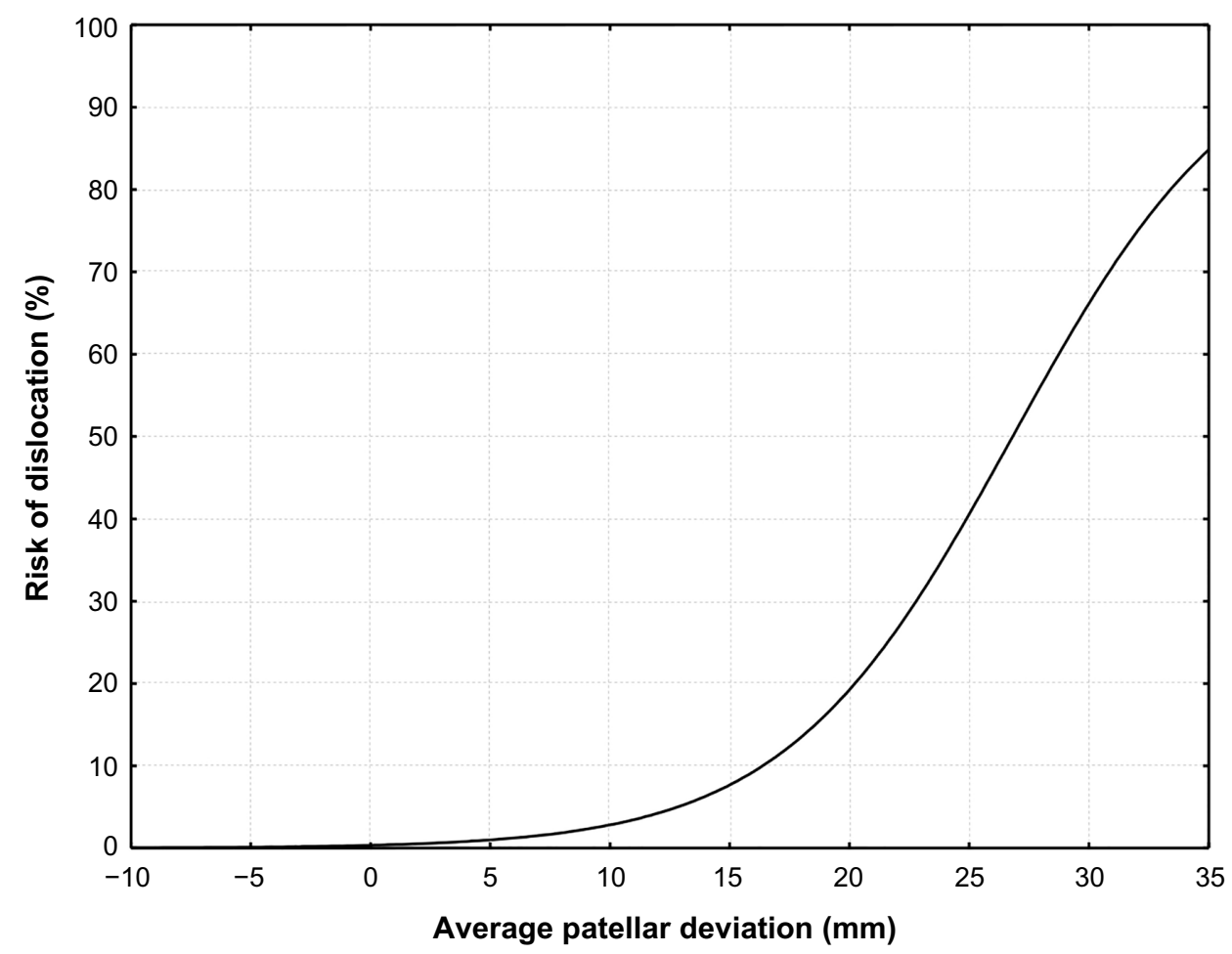

Figure 5 Risk of group 3 dislocations according to APD measured. The risk of dislocation increases significantly with measurements over 10 mm. When the APD value is $35 \mathrm{~mm}$, the risk increases by $85 \%$.

Abbreviation: APD, average patellar deviation.

and CT scans. This group of referenced values became widely known as the Lyon protocol. Nove-Josserand and Dejour ${ }^{34}$ added reference values for patellar tilt and deviation (subluxation) when the knees are relaxed or contracted, as well as mean values.

Given that the Lyon protocol is an accurate method with proven sensitivity and specificity in the analysis of patellar instability, we have chosen it as our guideline protocol for evaluation of patients with patellofemoral syndromes.

We observed a greater prevalence of women with patellar malalignment (58.7\% in group 3 and $64.7 \%$ in group 4$)$ in our study, which is consistent with other reports in the literature, ${ }^{22,35}$ except for those by Hughston ${ }^{23}$ and Reikeras and Hoiseth, ${ }^{37}$ who found no statistically significant difference in sex prevalence.

Rotational abnormalities of the lower limb, including femoral anteversion, knee rotation, external tibial torsion, and malleolar condylar angle, are considered possible etiologies for secondary disorders of the extensor apparatus, but there is no consensus in the literature as to whether there is a correspondence between these parameters and patellofemoral instability. ${ }^{7,15,17}$ Our results for femoral neck anteversion and malleolar condylar angle were higher in patients with potential or objective patellar instability than in groups 1 and 2 , but there was no statistically significant difference to support such differences as a primary etiology for patellar instability.

It is established that the basic factors predisposing patellar instability are trochlear dysplasia, lateralization of the tibial tuberosity, increased patellar height, and lateral patellar tilt and deviation. ${ }^{8,10,13,36,40}$

Trochlear dysplasia can be measured through the trochlear groove angle. In this study, this measurement was statistically significant for diagnosing patellar instability, but was not able to predict patellar dislocation. The literature reports regarding this matter are controversial. Some authors believe that there are no differences in results, regardless of which type of instability the patient has, ${ }^{21}$ while other studies report the importance of this method even to define surgical treatment for patellar dislocation. ${ }^{8,40}$

Increased lateralization of the tibial tuberosity can be evaluated by TTTG..$^{27,33}$ TTTG is an absolute value that does not take the patient's height and weight into account. In this study, TTTG was the most specific and sensitive method for detecting patellar instability when comparing groups 1 and 3. Further, this method was accurate for predicting the risk of patellar dislocation in patients with potential patellar instability.

To analyze patellar height in the 921 patients, the Insall-Salvatti method ${ }^{26,39}$ was applied in patients' CT scan 
tridimensional reconstruction with the knee in extension (relaxed and contracted) and 20 degrees of flexion. Our results showed this method to be a good option for diagnosing patellar instability, but not for predicting dislocation, which is in agreement with previous reports. ${ }^{8,40}$

Patellar tilting measurements in our control group showed small and insignificant differences with the knee extended or with a contracted quadriceps. When knees in this group were compared with those in groups 3 and 4, patellar tilt significantly increased $(P<0.05)$. When the same measurement was applied on a contracted quadriceps, there was a notable worsening of the patellofemoral alignment when compared with a relaxed quadriceps. The literature is controversial with regard to defining the actual need for an imaging study of the knee with a contracted quadriceps. Many studies have reported that muscle contraction can provide a more accurate imaging result in patients with patellofemoral malalignment, when compared with an imaging study with a relaxed muscle. ${ }^{19,20,28}$ Martínez et al consider two imaging studies to be unnecessary, and prefer a study with a relaxed muscle, since it is easier to perform. ${ }^{31}$

Patellar tilt is an objective flaw in the extensor apparatus and predicts patellar instability. In this regard, some authors have reported on the importance of the medial restrictors of the patella. ${ }^{9,19,30}$ An abnormality in the quadriceps muscle is not the only anatomical issue when there is dysplasia of the vastus medialis; it is a functional abnormality of the whole quadriceps compartment, with changes in both the vastus medialis and vastus lateralis muscles.

All CT measurements analyzed with relaxed and contracted quadriceps had variations in their values. Otherwise, mean patellar tilt values were the most sensitive and specific for evaluating this difference, and are in agreement with the results reported by Nove-Josserand and Dejour. ${ }^{34}$

After defining the most suitable diagnostic methods for detecting patellofemoral instability, we attempted to differentiate between patients who already had a patellar dislocation (group 4) and those with a high risk of dislocation (group 3). When logistic regression analysis was performed, TTTG, APH, APT, and APD were the most significant measurements with regard to predicting the probability of patellar dislocation, making it possible to create probability graphs. The most sensitive measurements were APT and APD, which is consistent with the finding of Nove-Josserand and Dejour that an increase in patellar tilt is a characteristic of patellofemoral instability and is not necessarily a consequence of dislocation. ${ }^{34}$

It is important to note that the patella behaves in a dynamic manner, so if the trochlear groove and TTTG are static on one side, which is necessary to stabilize the femur over the tibia, the ligamentous, muscular, and retinacular functions of the patella on the other side are of paramount importance to avoid or at least decrease the risk of dislocation after correct treatment.

The study has some limitations. We have a general population and a lack of interobserver and intraobserver reliability. Moreover, this study was cross-sectional in nature, and would have been better if performed in a prospective manner. Although not the aim of the study, we did not evaluate soft tissue factors, which can also increase the risk of dislocation. ${ }^{6,38}$

We conclude that quantification of deviation is important to plan treatment for patellofemoral imbalance in clinical practice. Our results allow us to qualify, quantify, and predict a possible patellar dislocation in patients with patellar malalignment. Trochlear groove angle, TTTG, APT and APH are the best tomographic tools available for diagnosis of patellar instability. To predict the probability of patellar dislocation, the most reliable measurements are TTTG, APH, APT and average patellar deviation.

\section{Disclosure}

The authors report no conflicts of interest in this work.

\section{References}

1. Aglietti P, Buzzi R, Insall JN. Disorders of the patellofemoral joint. In: Insall JN, Scott WN, editors. Surgery of the Knee. 3rd ed. Philadelphia, PA, USA: Churchill Livingstone; 2001.

2. Aulisa AG, Falciglia F, Giordano M, Savignoni P, Guzzanti V. Galleazi's modified technique for recurrent patella dislocation in skeletally immature patients. J Orthop Sci. 2012;17:148-155.

3. Biedert RM, Bachmann M. Anterior-posterior trochlear measurements of normal and dysplastic trochlea by axial magnetic resonance imaging. Knee Surg Sports Traumatol Arthrosc. 2009;17:1225-1230.

4. Blumensaat C, Ergenbn D. Patellofemoral disorders: physical and radiographic evaluation. Chir U Orthop. 1938;31:149-223.

5. Brattstrom $\mathrm{H}$. Shape of the intercondylar groove normally and in recurrent dislocation of patella: a clinical and x-ray anatomical investigation. Acta Orthop Scand. 1964;68:134-148.

6. Camp CL, Stuart MJ, Krych AJ, et al. The tibial tubercle-trochlear groove distance: author's response. Am J Sports Med. 2013;41: NP51-NP52.

7. Caton J, Deschamps G, Chambat P, Lerat JL, Dejour H. [Patella infera: a propos of 128 cases]. Rev Chir Orthop Reparatrice Appar Mot. 1982;68: 317-325. French.

8. Colvin AC, West RV. Patellar instability. J Bone Joint Surg Am. 2008; 90:2751-2762.

9. Conlan T, Garth WP, Lemons JE. Evaluation of the medial soft-tissue restraint of the extensor mechanism of the knee. J Bone Joint Surg. 1993;75:682-693.

10. Dejour D, Mercado J. [Lês Syndromes fémoro-patellaires. Diagnostic, facteurs de I'instabilité rotulienne et classification]. Médecins du Sport. 2006;78:15-22. French.

11. Dye SF, Campagna-Pinto D, Dye CC, Shifflett S, Eiman T. Soft-tissue anatomy anterior to the human patella. J Bone Joint Surg. 2003;85A: 1012-1017. 
12. Ficat RP. [Patellofemoral Pathology]. Paris, France: Masson et Luc; 1970. French.

13. Fithian DC, Neyret P, Servien E. Patellar instability: the Lyon experience. Tech Knee Surg. 2007;6:112-123.

14. Fithian DC, Paxton EW, Cohen AB. Indications in the treatment of patellar instability. J Knee Surg. 2004;17:47-56.

15. Fucentese SF, Schottle PB, Pfirrmann CW, Romero J. CT changes after trochleoplasty for symptomatic trochlear dysplasia. Knee Surg Sports Traumatol Arthrosc. 2007;15:168-174.

16. Galland $\mathrm{O}$, Walch $\mathrm{G}$, Dejour $\mathrm{H}$, et al. An anatomical and radiological study of the femoropatellar articulation. Surg Radiol Anat. 1990;12: 119-125.

17. Goutalier D, Bernageau J, Lecudonnec B. [Mesure de l'ecart tuberosité tibial antérieure-gorge de la trochlée TA-GT]. Rev Chir Orthop. 1978;64:423-428. French.

18. Grelsamer RP. Patellofemoral semantics. The Tower of Babel. The International Patellofemoral Study Group. Am J Knee Surg. 1997;10: 92-95.

19. Grelsamer RP, Klein JR. The biomechanics of the patellofemoral joint. J Orthop Sports Phys Ther. 1998;28:286-298.

20. Guzzanti V, Gigante A, Di Lazzaro A, et al. Patellofemoral malalignment in adolescents. Computerized tomographic assessment with or without quadriceps contraction. Am J Sports Med. 1994;22:55-60.

21. Haim A, Yaniv M, Dekel S, Amir H. Patellofemoral pain syndrome. Clin Orthop Relat Res. 2006;451:223-228.

22. Hallisey MJ, Doherty N, Bennett WF, Fulkerson JP. Anatomy of the junction of the vastus lateralis tendon and the patella. J Bone Joint Surg Am. 1987;69:545-549.

23. Hughston JC. Subluxation of the patella. J Bone Joint Surg Am. 1968;50A:1003-1026.

24. Hungerford DS, Barry M. Biomechanics of the patellofemoral joint. Clin Orthop Relat Res. 1979;144:9-15.

25. Inoue M, Shino K, Kirose H, Horibe S, Ono K. Subluxation of the patella. Computed tomography analysis of patellofemoral congruence. J Bone Joint Surg Am. 1988;70:1331-1337.

26. Insall J, Salvatti E. Patella junction in the normal knee joint. Radiology. 1971;101:101-104.

27. Jones RB, Bartlett EC, Vainright JR, Carroll RG. CT determination of tibial tubercle lateralization in patients presenting with anterior knee pain. Skeletal Radiol. 1995;24:505-509.
28. Kujala UM, Jaakkola LH, Koskinen SK, Taimela S, Hurme M, Nelimarkka O. Scoring of patellofemoral disorders. Arthroscopy. 1993;9:159-163.

29. Laurin CA, Dussault R, Levesque HP. The tangential x-ray investigation of the patellofemoral joint. Clin Orthop Relat Res. 1979;144:16-26.

30. Marczyk LRS, Gomes JLE. [Instabilidade femoropatelar: Conceitos atuais]. Rev Bras Ortop. 2000;35:275-281. Spanish.

31. Martinez S, Korobkin M, Fondren FB, Hedlund LW, Goldner JL. Diagnosis of patellofemoral malalignment by computed tomography. J Comput Assist Tomogr. 1983;7:1050-1053.

32. Merchant AC, Mercer RL, Jacobsen RH, Cool CR. Roentgenographic analysis of patellofemoral congruence. J Bone Joint Surg Am. 1974;56A:1391-1396.

33. Muneta T, Yamamoto H, Ishibashi T, Asahina S, Furuya K. Computerized tomographic analysis of tibial tubercle position in the painful female patellofemoral joint. Am J Sports Med. 1994;22:67-71.

34. Nove-Josserand L, Dejour H. [Quadríceps dysplasia and patellar tilt in objective patellar instability]. Rev Chir Orthop Reparatrice Appar. 1995;81:497-504. French.

35. Pozzi JFA, Konkewicz ER, Nora B. [Tratamento das instabilidades rotulianas]. Rev Bras Ortop. 1993;28:277-283. Spanish.

36. Redziniak DE, Diduch DR, Mihalko WM, et al. Patellar instability. Instr Course Lect. 2010;59:195-206.

37. Reikeras O, Hoiseth A. Patellofemoral relationships in normal subjects determined by computed tomography. Skeletal Radiol. 1990;19:591-592.

38. Sadoghi P, Camp CL, Stuart MJ, et al. The tibial tubercle-trochlear groove distance: letter to the editor. Am J Sports Med. 2013;41: NP51-NP52.

39. Schueda MA, Astur DC, Arliani GG. Comparative validation of the measure patellar height radiographic and tomography. Rev Bras Ortop. 2013;48:397-402.

40. White BJ, Sherman OH. Patellofemoral instability. Bull NYU Hosp Jt Dis. 2009;67:22-29.

41. Wiberg G. Roentgenographic and anatomic studies on the patellofemoral joint. With special reference to chondromalacia patella. Acta Orthop Scand. 1941;12:319-410.
Open Access Journal of Sports Medicine

\section{Publish your work in this journal}

Open Access Journal of Sports Medicine is an international, peer-reviewed, open access journal publishing original research, reports, reviews and commentaries on all areas of sports medicine. The manuscript management system is completely online and includes a very quick and fair peer-review system.

\section{Dovepress}

Visit http://www.dovepress.com/testimonials.php to read real quotes from published authors. 